\title{
Self-tuning PID Controller for Torque Tracking Control of Electrohydraulic Servo Systems
}

\author{
Lie $\mathrm{Yu}^{1,2}$, Jianbin Zheng ${ }^{1,2}$, Yang Wang ${ }^{1,2, *}$, Qiuzhi Song ${ }^{3}$ and Enqi Zhan ${ }^{1,2}$ \\ ${ }^{1}$ School of Information Engineering, Wuhan University of Technology, Wuhan, \\ China \\ ${ }^{2}$ Key Laboratory of Fiber Optic Sensing Technology and Information Processing, \\ Ministry of Education, Wuhan University of Technology, Wuhan, China \\ ${ }^{3}$ School of Mechatronical Engineering, Beijing Institute of Technology, Beijing, \\ China \\ E-mail:powerflow@whut.edu.cn
}

\begin{abstract}
Electrohydraulic servo systems (EHSS) have been widely used in industry and play an important role in the engineering applications. Proportional-integral-differential (PID) controller is utilized extensively to control the EHSS, but the tracking performance is limited using this approach, due to the nonlinear dynamics of EHSS. In this paper, we propose a self-tuning PID controller to improve the tracking performance of EHSS which is used to drive the One-Degree-Of-Freedom (1 DOF) rotary system. The gains of the proposed controller are adjustable parameters which are designed to vary within a predetermined range to eliminate the problems caused by the conventional PID controller. The simulation results show that the proposed self-tuning PID controller gives better tracking performances, such as less tracking lag and less mean absolute error (MAE), when compared with the conventional PID controller.
\end{abstract}

Keywords: Electrohydraulic Servo Systems; Tracking Performance; One-Degree-OfFreedom Rotary System; Conventional PID Controller; Self-tuning PID Controller

\section{Introduction}

Electrohydraulic servo systems (EHSS) have been widely used for their ability of high load efficiency, fast response, high tracking accuracy and big ratio of power to weight [1]. Typical applications of EHSS include turbine control, commercial aircraft, control of industrial robots, active suspension and automated manufacturing systems [2].

Based on the desired objectives, control strategies for EHSS are mainly classified as position control [3-6], velocity control [7] and force/torque control [8-9]. As to any one types of the control strategies, the controller selection will directly effect the tracking performance of EHSS. Generally, the conventional PID controller is most widely used due to its simple control structure, ease of design and low cost. However, large gains of the conventional PID controller result in undesirable oscillations, while small gains lead to big tracking lags and errors [10].

To overcome these drawbacks, we propose a self-tuning PID controller which takes use of adjustable PID gains. In addition, the adjustable PID gains vary within a predetermined range. The simulation results show that the proposed self-tuning PID controller provides less tracking lag and less mean absolute error (MAE) than the conventional PID controller. 


\section{Modeling of the Electrohydraulic Servo Systems}

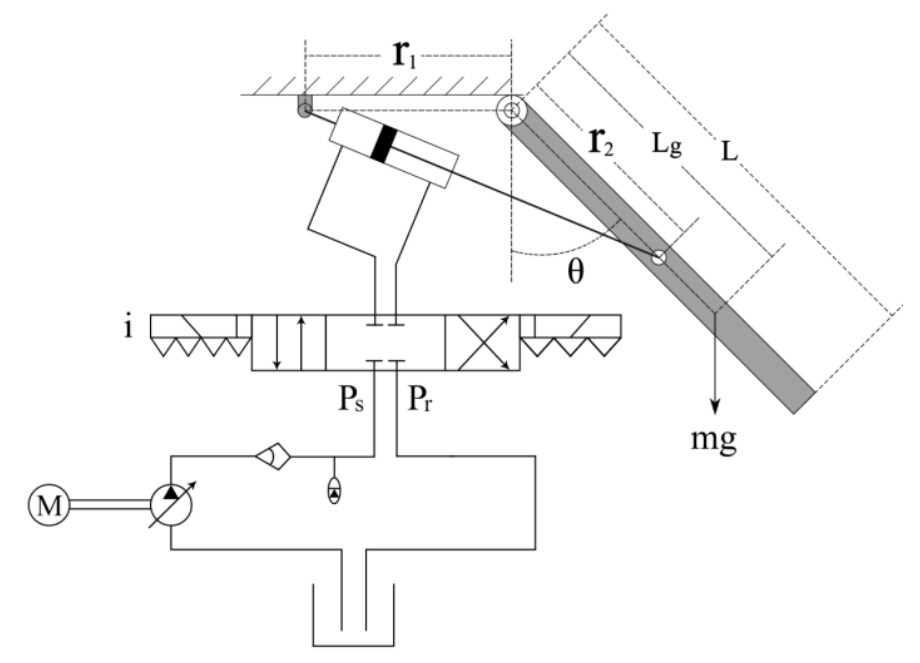

Figure 1. Schematic Diagram of the Electrohydraulic Servo System

As shown in Figure 1, the left part is the EHSS which mainly consists of a hydraulic and a servo valve, while the right part is the One-Degree-Of-Freedom (1 DOF) rotary system drive by the EHSS. Neglecting the frictional force, the dynamics of 1 DOF rotary system is considered as follows.

$$
J \ddot{\theta}=T_{L}-m g L_{g} \sin (\theta)
$$

where $J$ is the rotary inertia of the load; $\theta$ is the rotary angle; $T_{L}$ is the actuated torque; $m$ is the load mass; $g$ is the acceleration due to gravity; $L_{g}$ is the position of the center of the load mass.

However, the actuated torque $T_{L}$ can be acquired as

$$
\left\{\begin{array}{l}
T_{L}=\left(P_{1} A_{p 1}-P_{2} A_{p 2}\right) H \\
H=\frac{r_{1} r_{2} \cos (\theta)}{\sqrt{r_{1}^{2}+r_{2}^{2}+2 r_{1} r_{2} \sin (\theta)}}
\end{array}\right.
$$

where $P_{1}$ is the head-side pressure; $P_{2}$ is the rod-side pressure; $A_{p l}$ is the head-side area; $A_{P 2}$ is the rod-side area; $r_{1}$ and $r_{2}$ are the geometric length of the system as drawn in Figure 1.

The $A_{p 1}$ and $A_{P 2}$ can be calculated through the following formulas when the bore diameter $D_{l}$ and the rod diameter $D_{2}$ are acquired.

$$
\left\{\begin{array}{l}
A_{p 1}=\frac{\pi D_{1}^{2}}{4} \\
A_{p 2}=\frac{\pi\left(D_{1}^{2}-D_{2}^{2}\right)}{4}
\end{array}\right.
$$

As the the rod diameter $D_{2}$ is even less than the bore diameter $D_{1}$, the Equation (3) can be simplified as

$$
A_{p 2}=\frac{\pi\left(D_{1}^{2}-D_{2}^{2}\right)}{4} \approx \frac{\pi D_{1}^{2}}{4}=A_{p 1}
$$

Substituting the Equation (2) and Equation (4) into Equation. (1), the system model can be simplified as

$$
J \ddot{\theta}=P_{L} A_{p 1} H-m g L_{g} \sin (\theta)
$$

where $P_{L}=P_{1}-P_{2}$ is the load pressure of the dynamic actuator. 
As referred to [11-12], the external leakage of EHSS is extremely small and can be neglected. Then, the pressure dynamics of EHSS in both hydraulic chambers can be described as

$$
\left\{\begin{array}{l}
\dot{P}_{1}=\frac{\beta}{V_{1}}\left(-A_{p 1} v_{p}-C_{t} P_{L}+Q_{1}\right) \\
\dot{P}_{2}=\frac{\beta}{V_{2}}\left(A_{p 1} v_{p}+C_{t} P_{L}-Q_{2}\right)
\end{array}\right.
$$

where $\beta$ is the effective bulk modulus in the hydraulic chambers; $V_{1}$ and $V_{2}$ are the hydraulic chambers volumes and can be computed as $V_{l}=V_{0}+A_{p l} x_{p}, V_{2}=V_{0}-A_{p 1} x_{p} ; V_{0}$ is the chamber volume on the conditions that $x_{p}=0, V_{l}=V_{2}=V_{0}$ while $x_{p}$ is the hydraulic piston velocity; $C_{t}$ is the coefficient of the total internal hydraulic leakage; $Q_{l}$ is the supplied flow rate of the forward chamber, while $Q_{2}$ is the return flow rate of the return chamber. The $Q_{1}$ and $Q_{2}$ correlate with the servo-valve spool displacement $x_{v}$. Their relationships can be stated as

$$
\left\{\begin{array}{l}
Q_{1}=k_{q} x_{v}\left[s\left(x_{v}\right) \sqrt{P_{s}-P_{1}}+s\left(-x_{v}\right) \sqrt{P_{1}-P_{r}}\right] \\
Q_{2}=k_{q} x_{v}\left[s\left(x_{v}\right) \sqrt{P_{2}-P_{r}}+s\left(-x_{v}\right) \sqrt{P_{s}-P_{1}}\right]
\end{array}\right.
$$

where $P_{s}$ is the supply pressure of the fluid and $P_{r}$ is the return pressure. The $k_{q}$ is the valve discharge gain which can be described as

$$
k_{q}=C_{d} w \sqrt{\frac{2}{\rho}}
$$

where $C_{d}$ is the discharge coefficient, $w$ is the spool valve area gradient and $\rho$ is the density of hydraulic oil.

For Equation. (7), the function $s\left(x_{v}\right)$ is defined as

$$
s\left(x_{v}\right)= \begin{cases}1, & x_{v} \geq 0 \\ 0, & x_{v}<0\end{cases}
$$

However, due to the geometry structure, $x_{p}$ and $v_{p}$ can be calculated as

$$
\left\{\begin{array}{l}
x_{p}=\sqrt{r_{1}^{2}+r_{2}^{2}+2 r_{1} r_{2} \sin (\theta)}-L_{0}-x_{p 0} \\
v_{p}=\frac{2 r_{1} r_{2} \dot{\theta} \cos (\theta)}{\sqrt{r_{1}^{2}+r_{2}^{2}+2 r_{1} r_{2} \sin (\theta)}}
\end{array}\right.
$$

where $L_{0}$ is the cylinder dead length and $x_{p 0}$ is the piston position when the volumes are equal on both cylinder sides.

Generally, the input current (i) to the servo valve is directly proportional to the servovalve spool position $x_{v}$ such that it can be described as

$$
x_{v}=k_{c} i
$$

where $k_{c}$ is the positive electrical constant. Based on Equation (11), the function $s\left(x_{v}\right)$ can be equivalent as

$$
s\left(x_{v}\right)=s(i)
$$

Then, the Equation (7) can be rewritten as

$$
\left\{\begin{array}{l}
Q_{1}=g_{s} R_{1} i \\
Q_{2}=g_{s} R_{2} i
\end{array}\right.
$$

where $g_{s}$ is an integral parameter which can be describe as

$$
g_{s}=k_{q} k_{c}
$$


Meanwhile, the $R_{1}$ and $R_{2}$ in Equation (13) are defined as

$$
\left\{\begin{array}{l}
R_{1}=s(i) \sqrt{P_{s}-P_{1}}+s(-i) \sqrt{P_{1}-P_{r}} \\
R_{2}=s(i) \sqrt{P_{2}-P_{r}}+s(-i) \sqrt{P_{s}-P_{2}}
\end{array}\right.
$$

Based on the Equation (6) and Equation (13), the derivative of hydraulic load pressure can be rewritten as

$$
\dot{P}_{L}=\dot{P}_{1}-\dot{P}_{2}=\left(\frac{R_{1}}{V_{1}}+\frac{R_{2}}{V_{2}}\right) \beta g_{s} i-\left(\frac{1}{V_{1}}+\frac{1}{V_{2}}\right)\left(\beta C_{t} P_{L}+A_{p 1} v_{p}\right)
$$

Therefore, the time derivative of Equation (2) can be obtained as

$$
\dot{T}_{L}=A_{p 1}\left[\left(\frac{R_{1}}{V_{1}}+\frac{R_{2}}{V_{2}}\right) \beta g_{s} i-\left(\frac{1}{V_{1}}+\frac{1}{V_{2}}\right)\left(\beta C_{t} P_{L}+A_{p 1} v_{p}\right)\right] \frac{r_{1} r_{2} \cos (\theta)}{\sqrt{r_{1}^{2}+r_{2}^{2}+2 r_{1} r_{2} \sin (\theta)}}
$$

Before the controller design, the assumption must be followed that $P_{1}$ and $P_{2}$ are both bounded by $P_{s}$ and $P_{r}$, i.e. $0<P_{r}<P_{1}<P_{s}$ and $0<P_{r}<P_{2}<P_{s}$.

\section{Controller Design}

As the controller is designed to evaluate the torque tracking performance of EHSS, the actuated actual torque and the desired torque should be obtained before the controller design. In Eq. (13), the derivative of the actuated torque $T_{L}$ is acquired. However, the desired torque $T_{d}$ is not given to be a step or sinusoidal signal. In this paper, the system input is the rotary angle $\theta$ which is designed to be a sinusoidal signal.

$$
\theta(t)=\sin (2 \pi t)
$$

Therefore, the desired torque $T_{d}$ can be calculated using the Lagrange equation.

$$
T_{d}(t)=\left(m L_{g}^{2}+J\right) \ddot{\theta}(t)-m g L_{g} \sin (\theta(t))
$$

As described in [17], $T_{d}$ can also be named as computed virtual torque. Then, the controller design can be made at a sampling frequency of $f_{s}=1000 \mathrm{~Hz}$ to examine the behavior of the system.

\subsection{Design of the Conventional PID Controller}

The conventional PID controller can be constructed as follows.

$$
i(t)=k_{p} e(t)+k_{i} \int e(t) d t+k_{d} \frac{d e(t)}{d t}
$$

where $k_{p}, k_{i}$ and $k_{d}$ are the fixed proportional, integral and differential gains, respectively. $e(t)=T_{d}(t)-T_{L}(t)$ is the error between the desired torque and actuated torque of the system.

\subsection{Design of the Self-tuning PID Controller}

The design of the proposed controller is due to its gains which can be varied in changeable operating situations (i.e., the variation of torque error). When the torque error is large, the proportional gain can be selected to be a high value for better control effort. Similarly, when the torque error is small, the integral gain can be chosen to be a high value to overcome steady state error, and the differential gain can be also selected to be a high value for better system stability. The control law of the self-tuning PID controller can be written as: 


$$
\left\{\begin{array}{l}
i(t)=k_{p}(t) e(t)+k_{i}(t) \int e(t) d t+k_{d}(t) \frac{d e(t)}{d t} \\
k_{p}(t)=k_{p \max } f(t)+k_{p \min } g(t) \\
k_{i}(t)=k_{i \max } g(t)+k_{i \min } f(t) \\
k_{d}(t)=k_{d \max } g(t)+k_{d \min } f(t)
\end{array}\right.
$$

where

$$
\left\{\begin{array}{l}
f(t)=\frac{e^{k e(t)}-e^{-k e(t)}}{e^{k e(t)}+e^{-k e(t)}} \\
g(t)=1-f(t)
\end{array}\right.
$$

where $k$ is a constant to determines the rate of $k_{p}(t), k_{i}(t)$ and $k_{d}(t)$ varying between the maximum and minimum values. When the torque error $e(t)$ is large, the function $f(t)$ approaches $1 \quad(f(t) \rightarrow 1)$ and the function $g(t)$ approaches zero $(g(t) \rightarrow 0)$. Then, the proportional gain $k_{p}(t)$ approaches its maximum value $k_{p \max }\left(k_{p}(t) \rightarrow k_{p \max }\right)$ which is used to speed up the transient response and reduce the tracking lag. The integral gain $k_{i}(t)$ approaches its minimum value $k_{i \min }\left(k_{i}(t) \rightarrow k_{i \min }\right)$ which is used to eliminate the undesirable oscillations. The differential gain $k_{d}(t)$ approaches its minimum value $k_{d \min }$ $\left(k_{d}(t) \rightarrow k_{d \min }\right)$ which is used to reduce the rise time and tracking lag.

Similarly, when the torque error $e(t)$ is small, the function $f(t)$ approaches zero $(f(t) \rightarrow 0)$ and the function $g(t)$ approaches $1(g(t) \rightarrow 1)$. Then, the proportional gain $k_{p}(t)$ approaches its minimum value $k_{p \min }\left(k_{p}(t) \rightarrow k_{p \min }\right)$ which is used to eliminate the oscillations. The integral gain $k_{i}(t)$ approaches its maximum value $k_{i \max }\left(k_{i}(t) \rightarrow k_{i \max }\right)$ which is used to reduce the steady-state error. The differential gain $k_{d}(t)$ approaches its maximum value $k_{d \max }\left(k_{d}(t) \rightarrow k_{d \max }\right)$ which is used to reduce the adjustment time and enhance the system stability.

\section{Simulation Results}

\subsection{Selection of System Parameters}

In order to demonstrate the effectiveness of this system, the EHSS is simulated with the following nominal parameters: $J=0.96 \mathrm{~kg} \cdot \mathrm{m}^{2}, \quad L_{g}=0.24 \mathrm{~m}, \quad r_{l}=0.04 \mathrm{~m}, \quad r_{2}=0.27 \mathrm{~m}$, $P_{s}=2 \times 10^{6} \mathrm{~Pa}, \quad P_{r}=0.5 \times 10^{5} \mathrm{~Pa}, \quad L_{0}=0.1 \mathrm{~m}, \quad x_{p 0}=0.08 \mathrm{~m}, \quad L_{g}=0.37 \mathrm{~m}, \quad m=7 \mathrm{~kg}, \quad \beta=2 \times 10^{7} \mathrm{~Pa}$, $A_{p l}=1.77 \times 10^{-4} \mathrm{~m}^{2}, V_{0}=1.15 \times 10^{-4} \mathrm{~m}^{3}, C_{t}=8 \times 10^{-12} \mathrm{~m}^{5} \mathrm{~N}^{-1} \mathrm{~s}^{-1}, \mathrm{~g}_{\mathrm{s}}=3.97 \times 10^{-8} \mathrm{~m}^{4} \mathrm{~s}^{-1} \mathrm{~N}^{-1 / 2}, k=0.2$,

The gains of the conventional PID are chosen using Ziegler-Nichols method. Then, optimum gains of $k_{p}=0.5, k_{i}=3$ and $k_{d}=0.7 \times 10^{-3}$ are used. In addition, bigger gains of $k_{p}=1$, $k_{i}=4$ and $k_{d}=1 \times 10^{-3}$ are selected to test the tracking performance of the conventional PID. However, the gains of the self-tuning PID controller are chosen using the combination of the optimum gains and bigger gains that $k_{p \max }=1, k_{p \min }=0.5, k_{\text {max }}=4, k_{i \min }=3, k_{d \max }=1 \times 10^{-3}$ and $k_{d \min }=0.7 \times 10^{-3}$.

\subsection{Comparisons of the Conventional PID Controller and Self-tuning PID Controller}

The system simulation is implemented by Matlab based on the introduced EHSS model and 1-DOF rotary system model. The computer simulations are conducted using two types of controllers, such as the conventional PID controller and the self-tuning PID controller. Figure 2, Figure 3 and Figure 4 show the the tracking lag and mean absolute error (MAE) using the two types of controllers. 


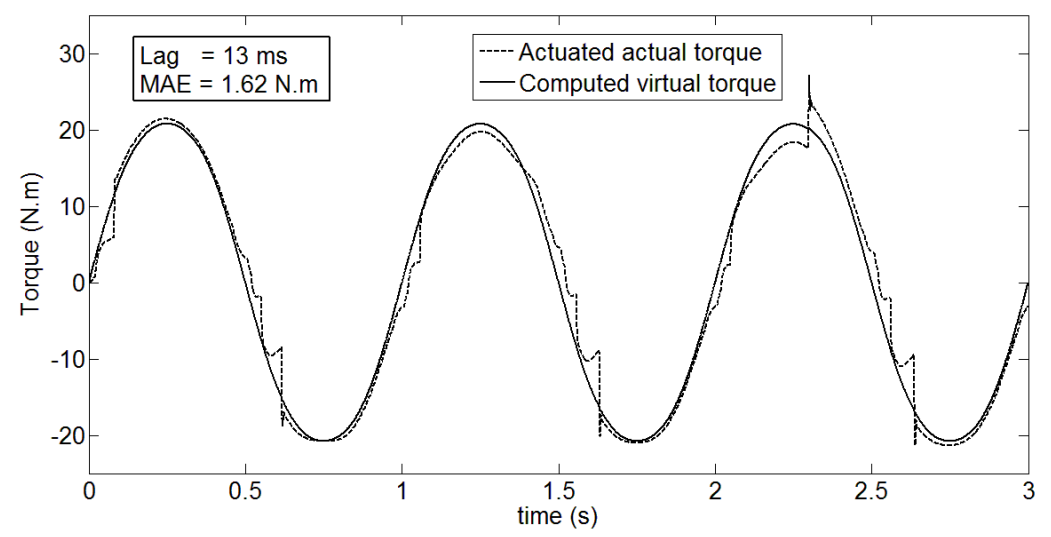

Figure 2. Tracking Performance of the Conventional PID Controller Using Optimum Gains

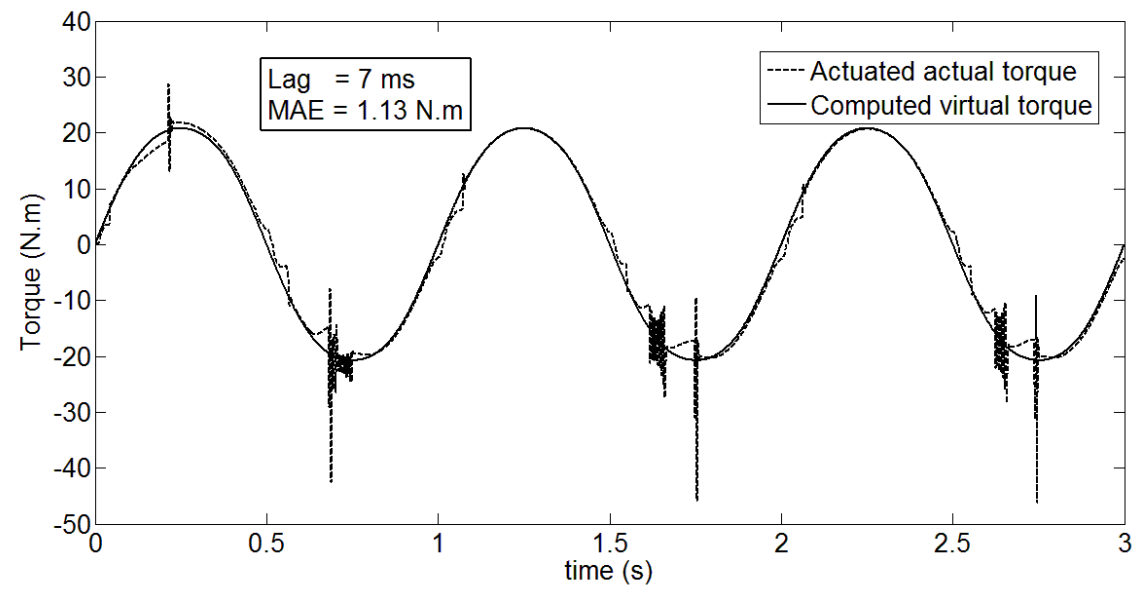

Figure 3. Tracking Performance of the Conventional PID Controller Using Bigger Gains

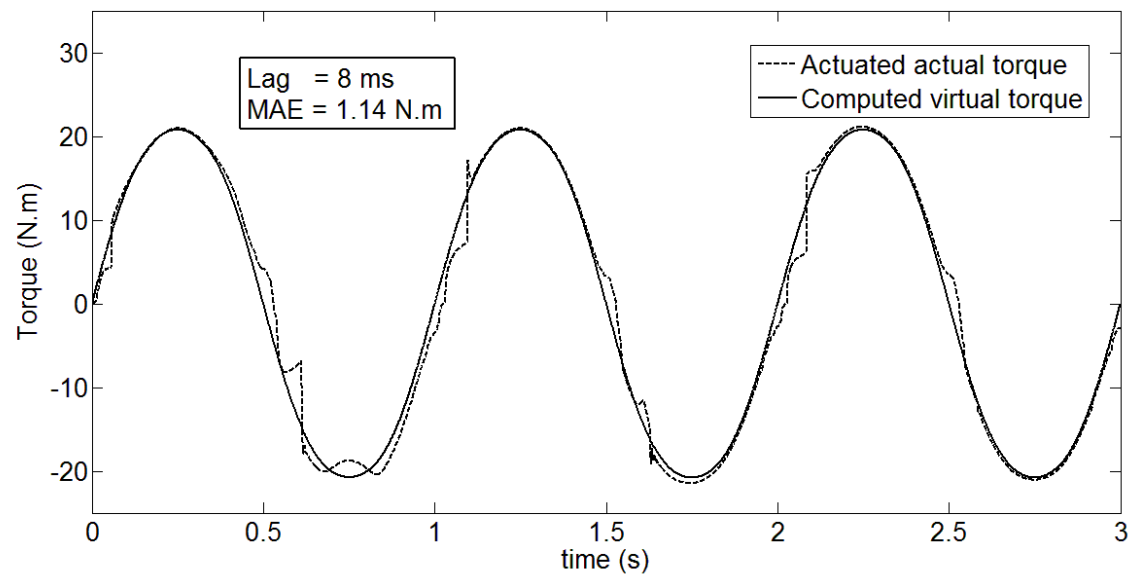

Figure 4. Tracking Performance of the Self-tuning PID Controller

The comparison between Figure 2 and Figure 4 describes that the tracking lag ( $8 \mathrm{~ms}$ ) of the self-tuning PID controller is less than that $(13 \mathrm{~ms})$ of the conventional PID controller 
using optimum gains. Meanwhile, the self-tuning PID controller obtains less MAE (1.14N.m) than the conventional PID controller (MAE=1.62N.m).

The comparison between Figure 3 and Figure 4 states that the conventional PID controller using bigger gains obtains better tracking performance (Lag=7ms and MAE $=1.13 \mathrm{~N} . \mathrm{m})$. However, the conventional PID controller receives oscillations in the control process while the self-tuning PID controller doesn't.

To sum up, the self-tuning PID controller not only eliminate the oscillations caused by the conventional PID controller using bigger gains, but also acquired less tracking lag and errors when compared with the conventional PID controller using optimum gains.

\section{Conclusion}

This paper has presented a self-tuning PID controller for EHSS. The 1 DOF rotary system as a test platform has been examined through simulations using the proposed selftuning PID. The gains of the proposed controller have been varied and tuned such that the EHSS receives better performance compared with the conventional PID controller in terms of trajectory tracking. It comes to a conclusion that the proposed self-tuning PID controller has given improved results when compared with the conventional PID controllers.

\section{References}

[1] J. C. Gao and P. D. Wu, "A fuzzy neural network controller in the electrohydraulic position control system", IEEE international conference on intelligent systems, (2003) October.

[2] H. A. Mintsa, R. Venugopal, J. P. Kenne and C. Belleau, "Feedback linearization-based position control of an electrohydraulic servo system with supply pressure uncertainty", IEEE transaction on control system technology., vol. 20, no. 4, (2012), pp. 1092-1099.

[3] P. Phakamach and C. Akkaraphong, "An optimal feedforward integral variable structure controller for the electrohydraulic position servo control systems", TENCON 2004. 2004 IEEE Region 10 Conference, (2004).

[4] E. Deticek, "An intelligent position control of electrohydraulic drive using hybrid fuzzy control structure", Proceedings of the IEEE International Symposium on Industrial Electronics., (1999).

[5] T. L. Chern and Y. C. Wu, "An optimal variable structure control with integral compensation for electrohydraulic position servo control systems", IEEE transaction on industrial electronics., vol. 39, no. 5, (1992), pp. 460-463.

[6] Q. P. Ha, Q. Nguyen, D. C. Rye and H. F. Durrant-Whyte, "Sliding mode control with fuzzy tuning for eletrohydraulic position servo system", 1998 second international conference on knowledge-based intelligent electronic system, (1998) April.

[7] G. Hasanifard, M. H. Zarif and A. A. Ghareveisi, "Nonlinear robust backstepping control of an electrohydraulic velocity servo system", Mediterranean conference on control and automation, (2007) July.

[8] S. Tafazoli, C. W. de Silva and P. D. Lawrence, "Tracking control of an elechydraulic manipulator in the presence of friction", IEEE Transactions on control system technology, (1998) May.

[9] S. Tafazoli, C. W. de Silva and P. D. Lawrence, "Friction estimation in a planar electrohydraulic manipulator", Proceedings of the American control conferences, Seatle, American, (1995) June.

[10] M. S. Zaky, "A self-tuning PI controller for the speed control of electrical motor drives", Electric Power Systems Research., (2015), pp. 293-303.

[11] J. Y. Yao, Z. X. Jiao and S. S. Han, "Friction compensation for low velocity control of hydraulic flight motion simulator: A simple adaptive robust approach", Chinese Journal of Aeronautics., (2013), pp. 814-822.

[12] J. Y. Yao, Z. X. Jiao and B. YAO, "Robust Control for Static Loading of Electro-hydraulic Load Simulator with Friction Compensation”, Chinese Journal of Aeronautics., (2012), pp. 954-962.

[13] J. C. Racine, "Control of a lower extremity exoskeleton for human performance amplification", University of California, Berkeley., (2003). 


\begin{abstract}
Authors
Lie $\mathbf{Y u}$, he is a Ph.D candidate from Wuhan University of technology, Wuhan, China. He received his B.E degree from Xidian University, in electronic information engineering, China, in 2009. He received his M.E degree from Wuhan University of Science and Technology, in control engineering, China, in 2011. His research interests mainly include the development of rehabilitation robots and its control application.
\end{abstract}

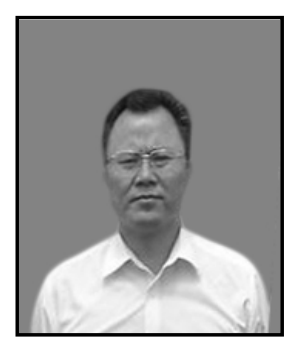

Jianbin Zheng, he is a professor at School of Information Engineering in Wuhan University of Technology, Wuhan, China. he received his B.E degree from University of Electronic Science and Technology of China, in communication and electronic system, China, in 1988, and the M.E degree from University of Electronic Science and Technology of China, in communication and electronic system, China, in 1991. His research interests focus on Information Processing, Pattern Recognition, Embedded System and Intelligent Control.

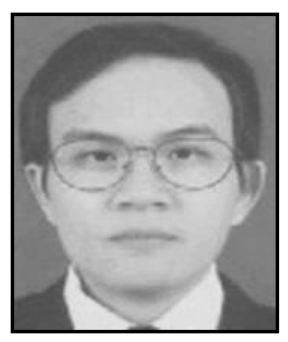

Yang Wang, he is presently working as an associate Professor in the School of Information Engineering, Wuhan University of Technology, Wuhan, China. He received his Ph.D degree in Wuhan University, Wuhan, China. His research areas are Robot Control and Pattern Recognition. *Corresponding author: Yang Wang, PhD, School of Information Engineering, Wuhan University of Technology, Jian Hu Campus, 122 Luo Shi Road, Hongshan District, Wuhan 430070, China, E-mail: powerflow@whut.edu.cn. 